To appear in: The Proceedings of the 6th International Symposium of MobileHCI 2004.

Ed. Brewster S., Dunlop M. Glasgow, UK: Springer. 426-430, 2004.

\title{
EMG as a Subtle Input Interface for Mobile Computing
}

\author{
Enrico Costanza, Alberto Perdomo, Samuel A. Inverso, Rebecca Allen \\ Liminal Devices, Media Lab Europe \\ Sugar House Lane, Bellevue, Dublin 8, Ireland \\ \{Enrico. Costanza, Alberto.Perdomo, Sam.Inverso, \\ Rebecca.Allen\}@medialabeurope.org \\ http://www. medialabeurope.org/
}

\begin{abstract}
Rather than merely imitating the desktop metaphor for mobile devices, new interface paradigms that take into account the particular characteristics of mobility, need to be developed. In this paper an input device based on the electromyographic (EMG) signal is proposed as a controller for mobile interaction. The interface can be considered subtle or intimate because individuals are able to interact privately without causing distraction to their immediate environment. The results from a preliminary study are presented to show the feasibility of the proposed system.
\end{abstract}

\section{Introduction}

With recent advances in microelectronics and display technology, current handheld devices such as mobile phones and PDAs are now powerful computing platforms that support network connectivity and embed colour touch screens. The user interfaces for these devices are generally derived from graphical interfaces for desktop computers using reduced versions of the keyboard and mouse. Rather than merely imitating the desktop metaphor for mobile devices new interface paradigms that take into account the particular characteristics of mobility, need to be developed. In a mobile context the user's attention should not be totally or even largely devoted to the computer interface. In addition, consideration should be given to the form of interaction in relation to the type of tasks that can be carried out in a mobile environment and its social acceptance. When the user is on the move or engaged socially, most of the computer interaction is of short duration. Often the user will be involved in simultaneous activities, e.g. talking, walking, and may be just checking for incoming messages in his mailbox.

A partial solution to the problems mentioned can be found in the use of output forms like audio [1,2] haptics [3] or graphic displays embedded in eyeglasses [4,5]. Different forms of input and output should be integrated in a multimodal interface to adapt to different tasks and situations. However, the interaction design for this type of systems constitutes an open challenge: the ideal mobile device should be 'hands-free' and 'eyes-free'. 
In this paper an input device based on the electromyographic (EMG) signal is proposed as a controller for mobile interaction. EMG allows the sensing of intentional muscle activity not necessarily related to articulation. In this way a class of "motionless" gestures can be defined to control applications on mobile devices. Such an interface can be considered subtle or intimate because individuals are able to interact privately without causing distraction to their immediate environment. This may improve the social acceptance of the interface.

\section{Related Work}

The electromyogram is an electrical signal generated by neuromuscular activity [6]. It can be recorded non-invasively using surface electrodes. Methods for effective recording and computer aided analysis of EMG signals have been the object of study in the field of biomedical engineering for the last three decades. EMG signals have been modelled as Gaussian like coloured zero mean noise [7]. Typical biomedical analysis involves envelope detection, energy measurement (directly related to the force) and frequency characterization. Research in this domain focuses on diagnosis applications of EMG.

Other studies in the domain of bioengineering have concentrated on the use of electromyographic signals for control of prosthesis, rehabilitation and computer interfaces for users with motor disabilities [8,9]. Beyond medical applications, EMG has been proposed for control of computer interfaces. Examples include interfaces for musical expression [10], controls for consumer electronics [11] and videogames [12]. All of these are based on EMG signals acquired from the forearm.

EMG based interfaces generally involve signal acquisition from a number of differential electrodes, signal processing (feature extraction) and real-time pattern classification. Classification methods based on both statistical and neural network approaches have been reported with satisfactory results. However, given the complexity of the task and the variability of the EMG signals [13] these systems usually require calibration for each user or training of the pattern recognition algorithms.

In a different fashion, but still in the context of HCI, EMG signals have been used in conjunction with other physiological signals (skin conductivity, blood pressure and respiration) to detect the affective state of the user [14].

A number of input interfaces based on gestures have been proposed for mobile and wearable computing. The most common approach is based on inertial sensors (accelerometers) worn by the user [1] or included on a PDA/mobile phone [15,16]; Rekimoto presented an interesting alternative based on capacitive sensing [17]. Some of the studies pose questions related to the social acceptance of the proposed gestures.

\section{Concept}

EMG can be used to sense isometric muscular activity [18]: the type of muscular activity that does not translate into movement. This feature makes it possible to define 
a class of subtle motionless gestures to control an interface without being noticed and without disrupting the surrounding environment. A simple generic controller in the form of an ancillary device is proposed. It can be placed on a muscle (for example the bicep) and activated by its contraction. When activation is detected, the controller sends a signal wirelessly to the main wearable processing unit, such as a mobile phone or PDA. The device is attached to an adjustable elastic band that can be hidden under clothes. Surface electrodes are integrated on the inside to acquire the signals. These are amplified, filtered and processed by integrated components. Compared to other EMG based controllers the approach proposed is to trade resolution (in terms of number of different gestures being recognized) for robustness and eliminate the need for calibration, yet keeping the computational cost to a minimum so that no external processing is required.

This simple controller can be used within a multimodal interface. In an example scenario the system has a display (eyewear or audio) capable of delivering high resolution information such as text (requiring a certain level of attention), as well as delivering low resolution peripheral cues (that do not require as much attention). Events such as new messages or phone calls generate cues. The user can react to cues by contracting the muscle, for example requesting more information about the event (e.g. the message subject or the caller ID). The peripheral cues can otherwise be ignored, if the user cannot afford to give attention to the computer. Using EMG, the user can react to the cues in a subtle way, without disrupting their environment and without using their hands on the interface.

\section{Preliminary study}

A preliminary study was carried out to explore the feasibility of the wearable EMG controller. A prototype was developed to record EMG data from moving subjects. The device acquires the physiological data and streams it wirelessly to a PC used for logging and offline analysis of the signals.

Three surface electrodes (input, reference and ground) are used to acquire the signal from the muscle. The input and reference signals are connected to an instrumentation amplifier and then filtered using a high-order low pass filter. An 8-bit microcontroller equipped with an integrated analogue to digital converter is used to sample the signal and transmit it to the PC using a Bluetooth ${ }^{\mathrm{TM}}$ module.

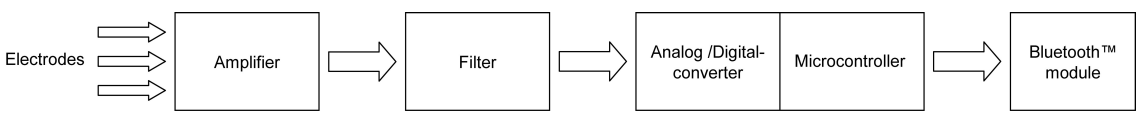

Fig. 1. Overview of the system hardware 
A group of 10 subjects ( 6 males, 4 females) between 25 and 33 years of age took part in the study. The subjects were informed of the purpose of the study and the function of the controller and then asked to "contract their muscle" in reaction to an audio stimulus. A total of 10 stimuli were presented aperiodically to each subject within 4 minutes. The sound was synchronized with the data logging, to facilitate the analysis. The electrodes $(\mathrm{Ag} / \mathrm{AgCl})$ were placed on the bicep and the device was worn on an armband. During the test the subjects were asked to move freely within a range of 10 meters to simulate realistic conditions.

A simple algorithm was designed to detect brief muscle contractions in the recorded EMG signal. The starting point for the design was the observation that in correspondence to a short muscle contraction the signal exhibits a peak, and that the duration of the peaks appeared to be consistent $(\approx 0.60-0.80 \mathrm{~s})$ across the different subjects even if no precise instruction on the duration of the contraction had been given. The signal was rectified and filtered with a moving average low pass filter tuned on the peak duration. Peaks are detected according to the following two conditions:

$$
\begin{gathered}
x_{n}-x_{n-T}<K_{1} \\
K_{2}<x_{n-T}-\frac{1}{4}\left(\sum_{i=1}^{4} x_{n-(i+1) T}\right)
\end{gathered}
$$

where $\mathrm{x}_{\mathrm{n}}$ denotes the current sample and T corresponds to a delay of $0.75 \mathrm{~s}$. The values of $\mathrm{K}_{1}$ and $\mathrm{K}_{2}$ were obtained by training the algorithm on a subset of the total data acquired. The training consisted in minimizing false positives and false negatives on the data collected from 4 of the subjects.

The muscle contraction was correctly detected in $84 \%$ of all cases ( 10 subjects). A number of 126 false detections occurred over the 40 minutes of signal recorded.

\section{Conclusion}

A subtle EMG based controller for mobile computing has been proposed. Results from a preliminary study show that even with simple processing techniques it is possible to detect brief muscle contractions in data acquired from moving subjects.

The results encourage further development of the interface. The signal processing and pattern recognition strategies should be improved to achieve higher accuracy. At the same time, the efficiency of the interface can be increased introducing feedback. The use of dry electrodes is being considered to promote user acceptance. Other muscles beside the bicep will be considered, including the combination of different ones. More in general, the authors plan to study the integration of the controller within a multimodal interface and the interaction design for the mobile context. Applications should be developed and user studies conducted to validate the general usability. 


\section{Acknowledgments}

The authors would like to thank the MindGames group, Matt Karau and Renee Hall at Media Lab Europe for their precious support.

\section{References}

[1] Lumdsen, J., Brewster, S.: A Paradigm Shift: Alternative Interaction Techniques for Use with Mobile \& Wearable Devices. In: Proceedings of 13th Annual IBM Centers for Advanced Studies Conference CASCON'2003, Toronto, Canada.

[2] Sawhney, N. Schmandt, C.: Nomadic Radio: Scaleable and Contextual Notification for Wearable Audio Messaging. In: Proceedings of the ACM SIGCHI Conference on Human Factors in Computing Systems, Pittsburgh.

[3] Toney, A., Dunne, L., Thomas, B., Ashdown, S.: A Shoulder Pad Insert Vibrotactile Display. In: Proceedings of the $7^{\text {th }}$ IEEE International Symposium on Wearable Computers (ISWC 2003), White Plains, New York.

[4] http://www.microopticalcorp.com/

[5] http://www.eyetap.org/

[6] De Luca, C.J.: Physiology and Mathematics of Myoelectric Signals. In: IEEE Transactions on Biomedical Engineering, Vol. BME-26, No.6, June 1979.

[7] De Luca, C.J.: Surface Electromyography: Detection and Recording. Whitepaper, DelSys Inc., 2002.

[8] Yamaguchi, H., Nishikawa, D., Yu, W., Maruishi, M., Yokoi, H., Mano, Y., Kakazu, Y.: EMG Control Switching System for FES. In: Proceedings of the 1999 IEEE International Workshop on Robot and Human Interaction, Pisa, Italy.

[9] Moon, I., Lee, M., Ryu, J., Mun, M.: Intelligent Robotic Wheelchair with EMG-, Gestureand Voice-based Interfaces. In: Proceedings of the 2003 IEEE/RSJ International Conference on Intelligent Robots and Systems, Las Vegas, NV.

[10] Tanaka, A., Knapp, R.B.: Multimodal Interaction in Music Using the Electromyogram and Relative Position Sensing. In: Proceedings of the 2002 Conference on New Instruments for Musical Expression (NIME-02), Dublin, Ireland.

[11] http://www.csl.sony.fr/ atau/gesture/

[12] Wheeler, K.R., Jorgensen, C.C.: Gestures as Input: Neuroelectric Joysticks and Keyboards. In: IEEE Pervasive Computing, April-June 2003 (Vol. 2, No. 2).

[13] Dubost, G., Tanaka, A.: A Wireless, Networked-based Biosensor Interface for Music. In: Proceedings of the ICMC 2002, Göteborg, Sweden.

[14] Healy, J., Picard, R.: Digital Processing of Affective Signals. In: Proceedings of ICASSP, Seattle, WA, 1998.

[15] Ängeslevä, J., O’Modhrain, S., Oakley, I., Hughes, S.: Body Mnemonics. In: Proceedings of Physical Interaction (PI03) - Workshop on Real World User Interfaces, a workshop at the Mobile HCI Conference 2003, Udine, Italy.

[16] Lehikoinen, J.: Virtual Pockets. In: Proceedings of the Fourth International Symposium on Wearable Computers (ISWC'00), Atlanta, Georgia, 2000.

[17] Rekimoto, J.: GestureWrist and GesturePad: Unobtrusive Wearable Interaction Devices. In: Proceedings of the $5^{\text {th }}$ IEEE International Symposium on Wearable Computers (ISWC 2001), Zurich, Switzerland.

[18] Greenman, P.E.: Principles of Manual Medicine. Publisher: Lippincott Williams \& Wilkins Publishers. 